\title{
Towards the improvement of cloud microphysical retrievals using simultaneous Doppler and polarimetric radar measurements
}

\author{
Y. Dufournet and H. W. J. Russchenberg \\ Remote Sensing of the Environment, Delft University of Technology, Postbus 5, 2600AA Delft, The Netherlands
}

Received: 30 September 2010 - Published in Atmos. Meas. Tech. Discuss.: 21 January 2011

Revised: 13 September 2011 - Accepted: 28 September 2011 - Published: 14 October 2011

\begin{abstract}
Radar-based retrievals are often employed to characterize the microphysical properties of cloud hydrometeors, i.e. their phases, habits, densities as well as their respective size and orientation distributions. These techniques are based on a synergetic use of different cloud observation sensor(s) and microphysical model(s) where the information extracted from both sensors and models is combined and converted into microphysical cloud properties. However, the amount of available information is often limited, which forces current microphysical retrieval techniques to base their algorithms on several microphysical assumptions which affect the retrieval accuracy.

By simultaneously combining Doppler and polarimetric measurements obtained from fully Doppler polarimetric radars, it is possible to create spectral polarimetric parameters. Although these parameters are easily contaminated with unwanted echoes, this work shows that, from a correct radar signal processing based on filtering and averaging techniques, spectral polarimetric parameters can be correlated to microphysical cloud properties. In particular, preliminary results suggest that particle orientations and habits can be determined from the sole use of such spectral polarimetric parameters. Therefore, such additional spectral polarimetric information offers an opportunity to improve current microphysical retrievals by reducing the number of microphysical assumptions in them.
\end{abstract}

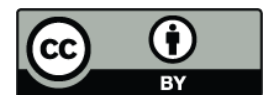

Correspondence to: Y. Dufournet (y.dufournet@tudelft.nl)

\section{Introduction}

There is a body of literature in atmospheric science that considers cloud microphysical parameterizations as one of the main sources of error in operational climate and weather forecast models (e.g. Cantrell and Heymsfield, 2005; Kärcher and Koop, 2005). Microphysical processes deal with small spatial and temporal scales (smaller than a few centimeters and up to an hour) which are not directly resolved in global circulation models (GCMs). Therefore, the parameterization of microphysical processes at scales resolved in GCMs (down to 100 kilometers and down to a few hours) is based on many assumptions which are currently not optimal at providing accurate descriptions of cloud microphysical properties. Parameters characterizing microphysical processes are cloud particle phases, their main orientations, their habits as well as their size distributions. A better understanding of such small scale processes and their characterization at large scales are of great importance in order to improve microphysical parameterizations. This can be achieved from the knowledge we gain from a proper use of cloud observation sensors (Shupe et al., 2008).

Radar sensors are commonly used to observe and retrieve microphysical properties within all types of cloud. An electromagnetic signal, transmitted from the radar through the atmosphere, is scattered by hydrometeors in a way that depends on the microphysical properties of the cloud. Thus, the backscattered signal (or better, the backscatter cross section) contains microphysical information of the hydrometeors being probed at different ranges along the radar line of sight. Most microphysical retrievals are based on the computation of the Doppler reflectivity spectrum from the

Published by Copernicus Publications on behalf of the European Geosciences Union. 
radar signal, which is defined as the distribution of hydrometeors radial velocities weighted by appropriate backscatter cross sections.

Typical radar-based retrievals only use the zero, first and second moments of the Doppler reflectivity spectrum, i.e. the reflectivity, the mean Doppler velocity and the Doppler width respectively. However, these radar quantities contain information coming from a mixture of several microphysical parameters (as mentioned above) which are difficult to differentiate without the synergetic use of other sensors and microphysical model information. Retrieval techniques are based on many type of synergies. For example, ice crystal size distributions and ice water contents within ice clouds can be retrieved from dual-frequency radar techniques (Hogan et al., 1999) or from radar-lidar observation algorithms (Donovan and Lammeren, 2001; Wang and Sassen, 2002; Tinel et al., 2005). Other synergies are also tested such as Radar Reflectivity - Infrared Radiometry (Mace et al., 1998) and, more recently, radar reflectivity - air temperature techniques (Hogan et al., 2006; Protat et al., 2007). Because the scope of application is often limited to specific cloud conditions (for example optically thin clouds when using radar-lidar techniques), it is finally worth mentioning that new types of algorithms treating radar-only, lidar-only and radar-lidar parts of the clouds are nowadays under study (Delanoe and Hogan, 2008). However, several output uncertainties arise in such types of retrievals due to sensor calibration issues, assumptions on non-retrieved microphysical properties (such as ice particle shape and orientation), simulation errors, and microphysical inhomogeneity within the large radar resolution volume being probed.

Assuming a small vertical wind speed, and taking into account the horizontal wind component, it is possible to associate particle fall velocities obtained by the radial velocity measurements with particle size distribution(s) of the cloud particle habit(s). Then, adding spectral information further improves the identification and partitioning of cloud particles present within the same radar resolution volume (Matrosov et al., 2002; Shupe et al., 2003; Deng et al., 2008; Delanoe et al., 2007). Additionally, particle shape/orientation can be linked to the polarimetric information coming from the medium being probed when radars are equipped with polarimetric capability (Russchenberg, 1992), which also enhances the microphysical characterization of cloud particles. In Matrosov et al. (2000), for example, polarimetric information is employed to infer the shape of cloud particles assuming spheroidal shapes and microphysical homogeneity within the radar resolution volume. Notwithstanding their limitations, these findings suggest that Doppler and polarimetric information significantly improve cloud retrieval algorithms mentioned previously by reducing the number of microphysical assumptions.

In this paper, it is proposed to go a step further by building spectral polarimetric parameters which simultaneously combine both polarimetric and Doppler information contained in fully polarimetric radar signals. It is expected that, based on these parameters, more cloud microphysical information is obtained, potentially reducing the number of assumptions employed in cloud retrievals. The use of spectral polarimetric parameters within cloud microphysical retrievals is therefore investigated in this paper.

In the following section, spectral polarimetric parameters are defined and associated with microphysical cloud properties. Small variation of particle motion and change in orientation produced by micro turbulence can make spectral polarimetric parameters very noisy. The second half of this section describes how to process them so that they become statistically coherent in space and time for their use in microphysical retrieval techniques. An example of a microphysical retrieval that uses spectral polarimetry is tested in Sect. 3, on several ice/mixed-phase cloud microphysical properties (habits and orientations). In the same section, an illustration based on a case study is provided in order to demonstrate the potentiality of such a technique under mixed-phase cloud conditions. Finally, in Sect. 4, a summary of the retrieval technique is presented.

\section{Spectral polarimetric parameters}

This section describes how spectral polarimetric parameters are defined and processed in this work. Some illustrations are provided using spectral polarimetric measurements obtained with the S-Band TARA radar (Transportable Atmospheric Radar - Heijnen et al., 2000) - see Fig. 1. The main TARA specifications used for microphysical measurements can be found in Table 1. Unlike most other atmospheric radars, TARA is based on the FM-CW principle (Frequency Modulated Continuous Wave). This technology brings the advantage of using a low transmitted power while possessing sensitivity comparable, or even higher, than pulse radar. It also provides better flexibility in terms of frequency modulation and sweep time (period of the frequency modulation), making the radar tunable for different spatial and time resolutions. TARA is composed of two large antennas, one continuously transmitting while the other continuously receives the backscattered signal. Both the transmitter and the receiver are equipped with polarizers that can change the polarization angle to either $0^{\circ}$ or $90^{\circ}$ (where $0^{\circ}$ corresponds to an horizontal polarization state). All polarimetric measurements can be performed quasi simultaneously. TARA is therefore a Doppler fully polarimetric atmospheric radar profiler and can combine several measurements in order to obtain the different spectral polarimetric parameters described in Sect. 2.1.

Doppler and polarimetric measurements are not optimal at the same elevation angle. On the one hand, Doppler information extracted from off vertical measurements is affected by the horizontal wind component which often has a higher order of magnitude compared to vertical wind and particle fall velocity combined, the latest being the parameter 
Table 1. Standard TARA specifications used for cloud microphysics measurements.

\begin{tabular}{|c|c|c|}
\hline Radar Parameter & value & comments \\
\hline central radar frequency & $3.298 \mathrm{GHz}$ & wavelength $=9.09 \mathrm{~cm}$ \\
\hline transmitted power & $\leq 100 \mathrm{~W}$ & $\begin{array}{l}\text { can be lowered in steps of } 10 \mathrm{~dB} \text { depending on the } \\
\text { meteorological situations }\end{array}$ \\
\hline Antenna elevation angle & $45^{\circ}$ & from the horizontal \\
\hline Measurements & value & comments \\
\hline range resolution & $10-30 \mathrm{~m}$ & \\
\hline Maximum unambiguous range & $5110-15330 \mathrm{~m}$ & Corresponding max. height $=3613-10840 \mathrm{~m}$ \\
\hline Doppler resolution & $\approx 3 \mathrm{~cm} \mathrm{~s}^{-1}$ & \\
\hline Maximum unambiguous Doppler velocity & $7.5 \mathrm{~m} \mathrm{~s}^{-1}$ (Nyquist velocity) & $22.5 \mathrm{~m} \mathrm{~s}^{-1}$ using the dealiasing technique \\
\hline
\end{tabular}

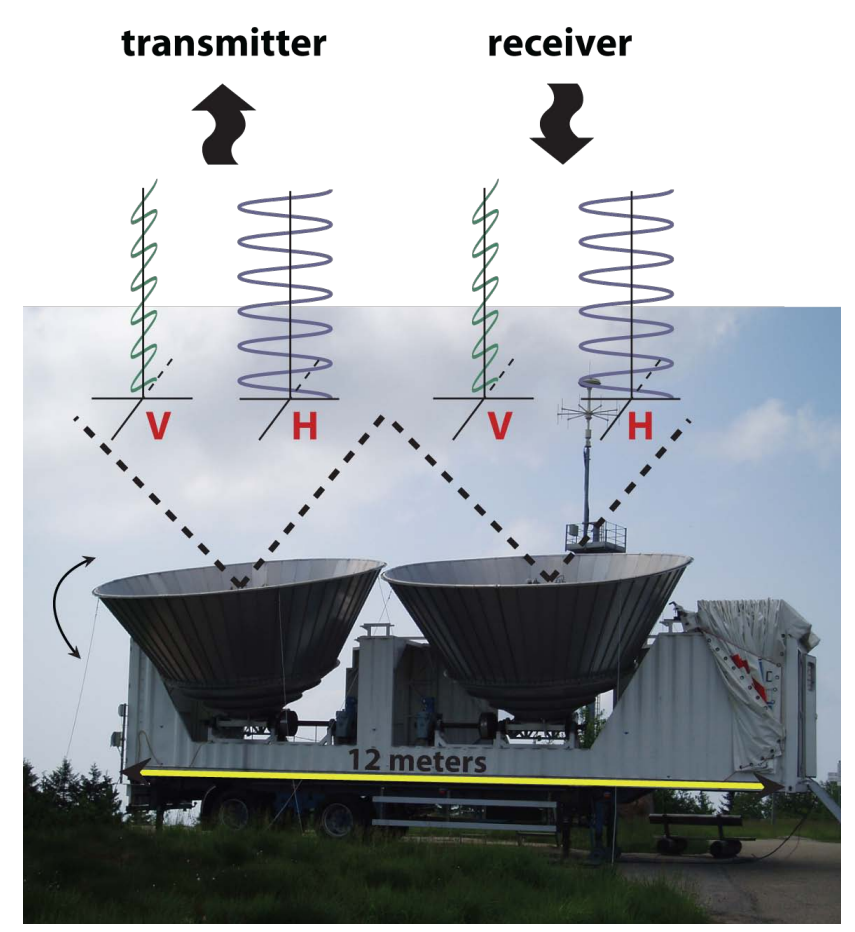

Fig. 1. The S-Band TARA radar. The transmitted and received signals are sequentially switched from vertical to horizontal polarization states in order to measure the full scattering matrix.

of interest within microphysical retrievals. The particle fall velocity derived from the Doppler spectrum is therefore optimally retrieved when performing vertically pointing measurements, the horizontal wind component being orthogonal to the radar line of sight and then negligible. On the other hand, polarization diversity measurements, i.e. polarimetric measurements which benefit from the non-spherical shape of falling oriented hydrometeors are most effective when performed close to the horizontal plane. As seen in Table 1, a trade-off at 45 degree elevation is taken in order to optimize the combination of both.

\subsection{Definition and microphysical interpretation}

Every spectral polarimetric parameter is based on the measured radial Doppler velocity spectrum. As previously mentioned, the radial Doppler velocity is a combination of the radial wind speed coupled with the intrinsic cloud particle fall velocity. On the one hand, the radial wind depends on the dynamic of the atmosphere and the radar elevation. On the other hand, the fall velocity spectrum is related to the microphysical properties of the ice crystals, i.e. particle size distribution, density, and particle area projected normal to the vertical airflow (Mitchell, 1996).

The primary description of how the radar target backscatters the transmitted radar signal is given by the so-called scattering matrix $\mathbf{S}$ (Bringi and Chandrasekar, 2001). For radars equipped with linear polarization, $\mathbf{S}$ is defined such that

$$
\left[\begin{array}{c}
\boldsymbol{E}_{\mathrm{h}}^{r} \\
\boldsymbol{E}_{\mathrm{v}}^{r}
\end{array}\right]=\frac{1}{r}\left[\begin{array}{cc}
S_{\mathrm{hh}} & S_{\mathrm{hv}} \\
S_{\mathrm{vh}} & S_{\mathrm{vv}}
\end{array}\right]\left[\begin{array}{c}
\boldsymbol{E}_{\mathrm{h}}^{i} \\
\boldsymbol{E}_{\mathrm{v}}^{i}
\end{array}\right]=\frac{1}{r} \mathbf{S}\left[\begin{array}{c}
\boldsymbol{E}_{\mathrm{h}}^{i} \\
\boldsymbol{E}_{\mathrm{v}}^{i}
\end{array}\right],
$$

with $\boldsymbol{E}^{r}=\boldsymbol{E}_{\mathrm{h}}^{r} \hat{\mathrm{h}}_{r}+\boldsymbol{E}_{\mathrm{v}}^{r} \hat{\mathrm{v}}_{r}$ the scattered field in the linear basis $\left(\hat{\mathrm{h}}_{r}, \hat{\mathrm{v}}_{r}\right)$. The subscripts $\mathrm{h}$ and $\mathrm{v}$ stand for the horizontal and vertical polarization states of the electric field, respectively while the superscripts $r$ and $i$ refer to the scaterred and incident waves, respectively. For a reciprocal medium, $S_{\mathrm{vh}}$ equals $S_{\mathrm{hv}}$, which reduces the scattering matrix to three elements. This reciprocity property holds for meteorological targets and quasi monostatic radars like TARA. The matrix elements can be obtained from any Doppler fully polarimetric atmospheric radar. From the Doppler information, the spectral scattering matrix elements, i.e. scattering matrix per Doppler bin, are computed. Each spectral polarimetric parameter is derived from the spectral scattering matrix elements and related to specific cloud properties.

The spectral horizontal/vertical reflectivity parameter $\boldsymbol{s} \boldsymbol{Z}_{\boldsymbol{X} \boldsymbol{X}}(v)$ (the subscripts $\boldsymbol{X}$ referring to either the vertical $(\boldsymbol{V})$ or horizontal $(\boldsymbol{H})$ polarization state of the receiver and the transmitter channels, respectively) is expressed as

$s \boldsymbol{Z}_{\boldsymbol{X} X}(v)=\left\langle S_{X X}(v) S_{X X}^{*}(v)\right\rangle$, 
where $<>$ denotes the time average of the Doppler spectra and $v$ represents each radial Doppler velocity bin. In particular, $\boldsymbol{s} \boldsymbol{Z}_{\boldsymbol{X} \boldsymbol{X}}(v)$ is related to the particle size distribution of the medium being probed.

The spectral differential reflectivity ratio $s Z_{D R}(v)$ is defined as the ratio between the two copolar signals, $s \boldsymbol{Z}_{\boldsymbol{H} \boldsymbol{H}}(v)$ and $s \boldsymbol{Z}_{V V}(v)$, such that

$\boldsymbol{s} \boldsymbol{Z}_{\boldsymbol{D} \boldsymbol{R}}(v)=\frac{\boldsymbol{s} \boldsymbol{Z}_{\boldsymbol{H} \boldsymbol{H}}(v)}{\boldsymbol{s} \boldsymbol{Z}_{\boldsymbol{V} \boldsymbol{V}}(v)}=\frac{\left\langle S_{\mathrm{hh}}(v) S_{\mathrm{vV}}^{*}(v)\right\rangle}{\left\langle S_{\mathrm{vv}}(v) S_{\mathrm{vV}}^{*}(v)\right\rangle}$.

The main advantage of this parameter is that it is independent of any absolute radar calibration. It is, above all, linked to the shape and orientation of the hydrometeors, a.g. their axis ratio assuming spheroidal shapes. Differences in some simulated $s Z_{D R}$ outputs are illustrated in Fig. 2 for different spheroidal particle shapes and orientations present in the radar resolution volume and for different particle microphysical properties. As mentioned previously, optimum $s Z_{D R}$ measurements are obtained at a 45 degrees elevation angle in order to benefit from both, the polarization diversity measurements from non-spherical hydrometeors, and the falling particle velocity information obtained from the Doppler measurements. Still, typical $\boldsymbol{s} \boldsymbol{Z}_{\boldsymbol{D} \boldsymbol{R}}(v)$ values lower than $\pm 2 \mathrm{~dB}$ are mostly found within cloud observation when used in radar profiling techniques which makes $s Z_{D R}(v)$ strongly affected by noise.

The ratio between the cross-polar and the co-polar spectral reflectivity is referred as the linear depolarization ratio $\boldsymbol{s} \boldsymbol{L}_{\boldsymbol{D} \boldsymbol{R}, \boldsymbol{X}}(v), \boldsymbol{X}$ being either the vertical or horizontal polarization state of the copolar reflectivity signal:

$\boldsymbol{s} \boldsymbol{L}_{\boldsymbol{D} \boldsymbol{R}, \boldsymbol{X}}(v)=\frac{\boldsymbol{s} \boldsymbol{Z}_{\mathrm{hv}}(v)}{\boldsymbol{s} \boldsymbol{Z}_{\boldsymbol{X} \boldsymbol{X}}(v)}=\frac{\left\langle S_{\mathrm{hv}}(v) S_{\mathrm{hv}}^{*}(v)\right\rangle}{\left\langle S_{\mathrm{xx}}(v) S_{\mathrm{xx}}^{*}(v)\right\rangle}$.

$\boldsymbol{s} \boldsymbol{L}_{\boldsymbol{D} \boldsymbol{R}, \boldsymbol{x}}(v)$ is mainly related to the tumbling effect of falling cloud particles. Note that this parameter has also been used to characterize particle habits within ice clouds (Matrosov et al., 2000).

The last spectral parameter, the spectral complex copolar cross-correlation coefficient $\boldsymbol{s} \rho_{\mathbf{c o}}(v)$, is defined as

$\boldsymbol{s} \rho_{\mathbf{c o}}(v)=\frac{\left\langle S_{\mathrm{hh}}(v) S_{\mathrm{vV}}^{*}(v)\right\rangle}{\sqrt{\left\langle\left|S_{\mathrm{hh}}\right|^{2}\right\rangle\left\langle\left|S_{\mathrm{vv}}\right|^{2}\right\rangle}}$.

As shown in Eq. (5), the spectral complex copolar crosscorrelation coefficient is associated with the correlation between the copolar horizontal and vertical spectra. Battaglia et al. (2001) demonstrated that $\rho_{\mathrm{co}}$, and therefore $\boldsymbol{s} \rho_{\mathbf{c o}}(v)$, is sensitive to the particle habit as for $\boldsymbol{s} \boldsymbol{L}_{\boldsymbol{D} \boldsymbol{R}, \boldsymbol{x}}(v)$.

\subsection{Standard spectral polarimetric processing}

The radar signal coming from the TARA receiver can be depicted as a mixture of wanted echoes (from meteorological targets), unwanted echoes (clutter), and noise. Standard spectral polarimetric processing steps are first applied based on the work of Unal and Moisseev (2004) and Unal (2009). This first standard processing uses spectral polarimetric techniques in order to strongly reduce part of the spectrum having non-meteorological spectral polarimetric properties and correct for possible spectrum aliasing. It guarantees an optimum trade-off by keeping most of the atmospheric echoes while removing as much noise and clutter as possible for any atmospheric situations. An illustration of this processing is provided in Fig. 3a-c.

\subsubsection{Smoothing}

The Doppler spectra computed from the raw signal are very noisy (see Fig. 3a), which make them difficult to study. The signal is first smoothed in order to remove most of the statistical variations while keeping the main slope of each spectrum (see Fig. 3a).

\subsubsection{Correction for spectral aliasing and non-simultaneous polarimetric measurements}

For the TARA radar, a sequence of measurements at different polarization settings is used in order to obtain all the spectral scattering matrix elements. Increasing sequence length consequently reduces the maximum unambiguous Doppler velocity of the radar. For some atmospheric situations with strong convection, this can lead to Doppler spectrum aliasing. Moreover, the target's dynamical properties also affect the polarimetric accuracy of the radar data because of the non-simultaneity of the measurements carried out at different polarization settings. The data are, therefore, corrected for spectral aliasing and non-simultaneous polarimetric measurement using the method of Unal and Moisseev (2004). A consequence of these corrections is also the reduction of noise and clutter echoes, which are not satisfying the spectral polarimetric signature expected from meteorological targets. Such unwanted echoes are indeed reallocated to a different Doppler velocity interval than the one where meteorological echoes are present (as can be seen in Fig. $3 b$ for the signal present below $-8 \mathrm{~m} \mathrm{~s}^{-1}$ or above $+8 \mathrm{~m} \mathrm{~s}^{-1}$.

\subsubsection{Double $s L_{D R}$ Clipping}

Polarimetric filtering using thresholding techniques is applied on the spectral linear depolarization ratios, $\boldsymbol{s} \boldsymbol{L}_{\boldsymbol{D} \boldsymbol{R}, \boldsymbol{H}}(v)$ and $\boldsymbol{s} \boldsymbol{L}_{\boldsymbol{D} \boldsymbol{R}, \boldsymbol{V}}(v)$, based on the work of Unal (2009). For both, values above $-5 \mathrm{~dB}$ are considered as non-meteorological echoes and are discarded from the Doppler spectra. Figure $3 \mathrm{c}$ shows an example of the $\boldsymbol{s} \boldsymbol{Z}_{\boldsymbol{V} \boldsymbol{V}}$ spectra obtained after thresholding on $\boldsymbol{s} \boldsymbol{L}_{\boldsymbol{D} \boldsymbol{R}, \boldsymbol{V}}(v)$ and $\boldsymbol{s} \boldsymbol{L}_{\boldsymbol{D} \boldsymbol{R}, \boldsymbol{H}}(v)$. In this specific case, the velocity interval where meteorological echoes are present is constrained between about $-9.7 \mathrm{~m} \mathrm{~s}^{-1}$ and $-3.6 \mathrm{~m} \mathrm{~s}^{-1}$. 

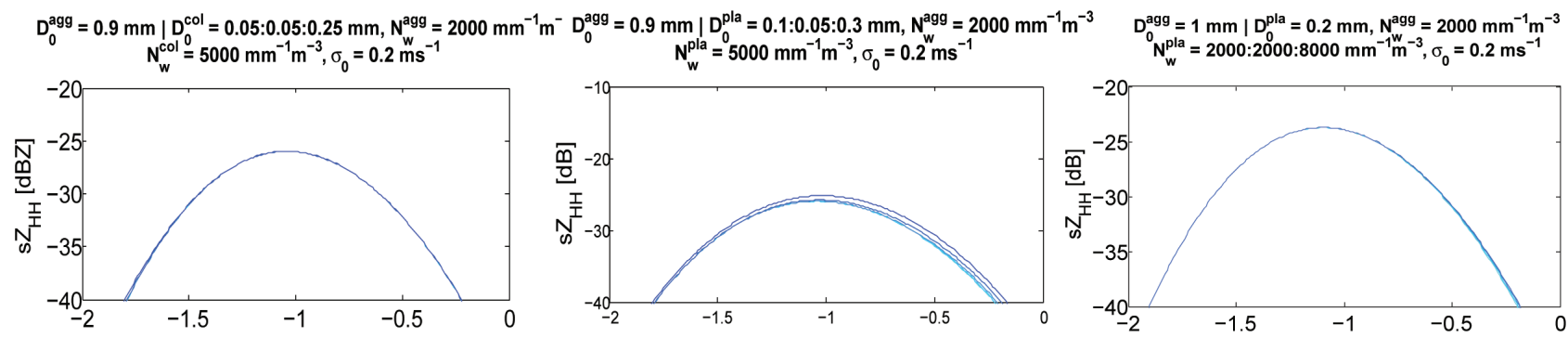

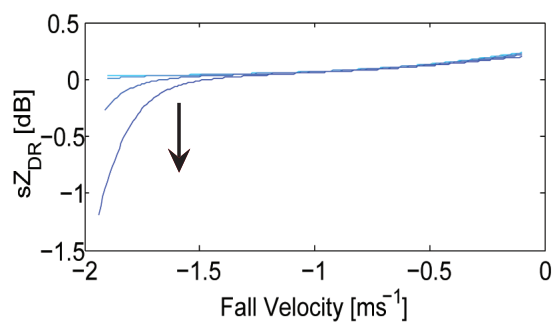

(a)

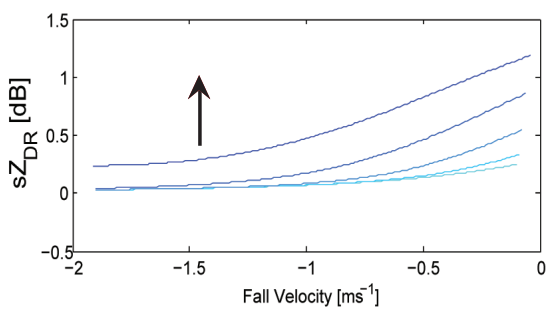

(b)

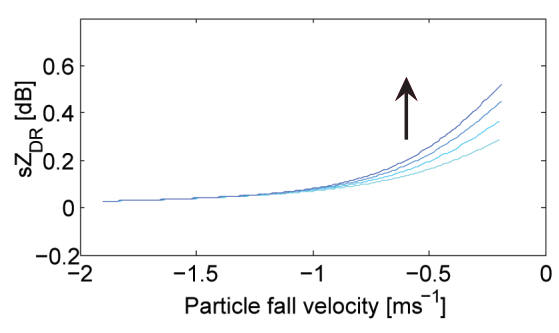

(c)

Fig. 2. Simulations of $s \boldsymbol{Z}_{\boldsymbol{H} \boldsymbol{H}}(v)$ and $\boldsymbol{s} \boldsymbol{Z}_{\boldsymbol{V} V}(v)$ obtained for different microphysical configurations. For all simulations, a mix of two particle types is considered. All particle shapes are approximated with spheroids and they all follow an exponential distribution. $D_{0}$ corresponds to the Median Volume Diameter, $N_{\mathrm{W}}$ is the intercept parameter (related to the particle concentration) and $\sigma_{0}$ is the spectral broadening parameter (related to the atmospheric turbulence within the volume considered). (a) Mixing of aggregates and columns obtained for an increase of the column crystals diameter. (b) Mixing of aggregates and plates obtained for an increase of the plate crystals diameter. (c) Mixing of aggregates and plates obtained for an increase of the plate crystals concentration.

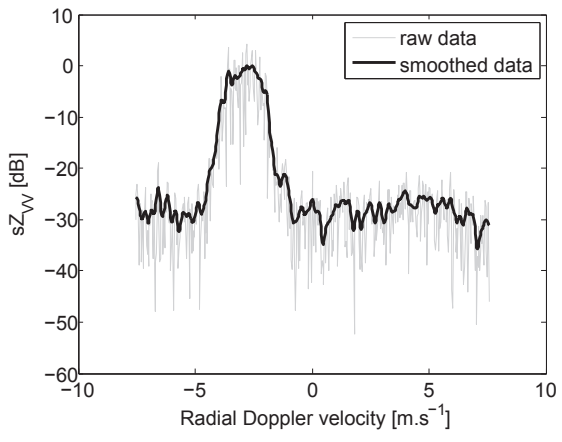

(a)

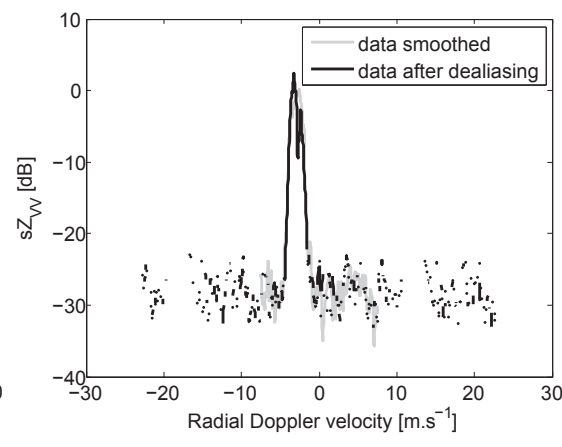

(b)

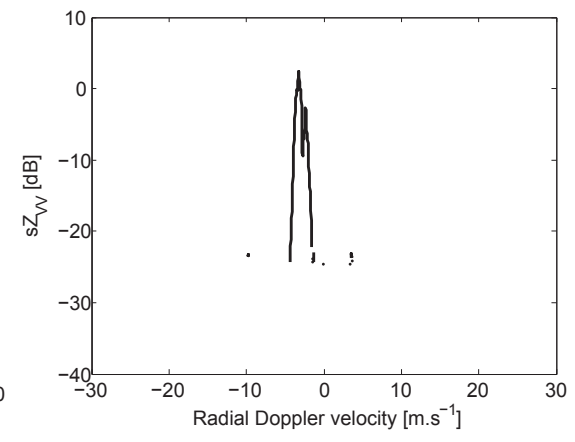

(c)

Fig. 3. TARA processing steps performed on $s Z_{\boldsymbol{V} V}(v)$ : (a) raw data and smoothed data, (b) correction for spectral aliasing and nonsimultaneous polarimetric measurements (the smoothed data from Fig. $3 \mathrm{a}$ is added in order to correctly see the changes performed after dealiasing) and (c) double $\boldsymbol{s} \boldsymbol{L}_{\boldsymbol{D} \boldsymbol{R}, \boldsymbol{x}}$ clipping. After (c), only a few noise data (dots in the spectra) are kept.

\subsection{Additional processing applied for microphysical retrievals}

The additional processing ensures a total noise and spectral fluctuation removal (which have not been removed from the standard processing) in order to use spectral polarimetry for cloud microphysical retrievals where high correlation between spectra in height and time is required. This additional processing is still in an experimental phase and is therefore first tested on high signal-to-noise-ratios in order to satisfy current retrieval techniques which are based on second order polynomial approximation (as described in Sect. 3).

After applying all the processing steps mentioned in Sects. 2.2.1-2.2.3, $\boldsymbol{s} \boldsymbol{Z}_{\boldsymbol{D} \boldsymbol{R}}(v)$ still remains affected by strong signal fluctuations and remaining clutter. In order to improve microphysical retrieval techniques based on spectral polarimetric parameters, further filtering is applied to $s \boldsymbol{Z}_{\boldsymbol{D} \boldsymbol{R}}(v)$ using additional clipping and statistical estimators. 


\subsubsection{Additional clipping}

The threshold values obtained for this additional clipping are obtained from a sensitivity analysis performed on the spectral polarimetric parameters. This analysis was carried out over a set of different ice/mixed-phase clouds probed with the TARA radar. It was found that, within ice cloud systems (precipitation and ice/mixed-phase clouds), the clipping on $\boldsymbol{s} \boldsymbol{L}_{\boldsymbol{D} \boldsymbol{R}, \boldsymbol{H}}(v)$ and $\boldsymbol{s} \boldsymbol{L}_{\boldsymbol{D} \boldsymbol{R}, \boldsymbol{V}}(v)$ could be rescaled to $-15 \mathrm{~dB}$ without affecting the meteorological echoes.

$\boldsymbol{s} \boldsymbol{Z}_{\boldsymbol{D R}}(v)$ within microphysical retrievals requires a very high correlation between the Doppler spectra obtained in $H H$ and $V V$ polarization states and, therefore, a total noise and clutter removal is needed. The remaining noise and clutter still present in the Doppler signal can be removed by an additional clipping based on spectral polarimetric correlation, using the spectral copolar cross-correlation coefficient $\boldsymbol{s} \rho_{\mathbf{c o}}(v)$. Within ice clouds, Battaglia et al. (2001) simulated different polarimetric parameters for different ice particle habits and radar elevations. At 45 degrees elevation angle (standard TARA elevation when working with spectral polarimetry), typical $\boldsymbol{s} \rho_{\text {co }}$ values above 0.9 were simulated. In this work, clipping is performed at $s \rho_{\mathbf{c o}}(v)=0.94$ in order to discard the remaining noise echoes.

Dealiasing, as described in Sect. 2.2.2, can produce an overestimation of $s \rho_{\mathbf{c o}}(v)$ in the remaining noise data region inherent in the cross-correlation computation (Eq. 5). This is not the case when looking at the variation of $s \rho_{\mathrm{co}}(v)$ on consecutive Doppler bins (in consecutive velocities at one specific time), i.e. $\Delta s \rho_{\mathrm{co}}(v)$. Thus, a third filtering is applied in parallel on $\boldsymbol{\Delta} \boldsymbol{s} \rho_{\mathbf{c o}}(v)$, values above 0.01 being removed from the spectra. In conclusion, only part of the Doppler spectrum satisfying the three thresholds on $\boldsymbol{s} \boldsymbol{L}_{\boldsymbol{D} \boldsymbol{R}, \boldsymbol{X}}(v), \boldsymbol{s} \rho_{\mathbf{c o}}(v)$ and $\Delta s \rho_{\text {co }}(v)$ is kept. An example of such additional clipping is provided in Fig. 4. For this specific example, the Doppler velocity interval of useable data is further constrained from $-4 \mathrm{~m} \mathrm{~s}^{-1}$ to $-1.8 \mathrm{~m} \mathrm{~s}^{-1}$.

\subsubsection{Additional averaging}

This use of additional Doppler velocity clipping doesn't remove the statistical variations present in the $\boldsymbol{s} \boldsymbol{Z}_{\boldsymbol{D} \boldsymbol{R}}$ Doppler interval where meteorological targets contribute. These fluctuations arise from the dynamical and microphysical inhomogeneities of the atmosphere. Such variations can produce incoherent behaviors across consecutive spectra in time if the Doppler spectra are not averaged. By averaging over time a number $N_{\text {av }}$ of initial non-averaged $\boldsymbol{s} \boldsymbol{Z}_{\boldsymbol{D} \boldsymbol{R}}$ spectra, it is possible to remove higher-order variations. At each range and time, the number of initial spectra to average (corresponding to a specific averaging time) is determined. It is based on a statistical analysis of $s Z_{D R}$ slopes, i.e. the main trend within each $s \boldsymbol{Z}_{\boldsymbol{D} \boldsymbol{R}}$ spectrum which is obtained from a second order polynomial fit in order to reduce the higher-order fluctuations of the signal. For the analysis, a set of Doppler spectra is created at each time and range bins of the reflectivity profile. Each set is composed of 15 consecutive spectra being averaged from the same initial time over an increasing averaging time (from $1.5 \mathrm{~s}$ to $22.5 \mathrm{~s}$ ). For the sake of clarity, the spectrum $1\left(N_{\mathrm{av}}=1\right)$ is not averaged (initial $1.5 \mathrm{~s}$ resolution) and the spectrum $15\left(N_{\mathrm{av}}=15\right)$ is averaged to a maximum time amount of $22.5 \mathrm{~s}$. The analysis is performed on each spectrum as well as its parabolic fit. Two different statistical parameters, the standard deviation std and the correlation factor Rd, are used as described below.

\section{The Standard deviation Std}

The parabolic fit is first used to compute the standard deviation at each averaging step,

$$
\operatorname{std}=\frac{\sqrt{\frac{1}{M} \sum_{v \min }^{v \max }\left|s \boldsymbol{Z}_{\boldsymbol{D R}}(v)-\boldsymbol{s} \boldsymbol{Z}_{\boldsymbol{D} \boldsymbol{R}}^{\mathrm{fit}}(v)\right|^{2}}}{\sqrt{\frac{1}{M} \sum_{v \min }^{v \max }\left|\frac{s \boldsymbol{Z}_{\boldsymbol{D} \boldsymbol{R}}(v)+s \boldsymbol{Z}_{\boldsymbol{D} \boldsymbol{R}}^{\mathrm{fit}}(v)}{2}\right|^{2}}},
$$

where $M$ corresponds to the total number of Doppler velocity bins and $s Z_{D R}^{\text {fit }}(v)$ is the parabolic fit of each averaged $s Z_{D R}$ spectrum (Fig. 5a). Std is related to the amplitude of the $s \boldsymbol{Z}_{\boldsymbol{D} \boldsymbol{R}}$ fluctuations. An example of std evolution for an increasing averaging time is provided in Fig. $5 b$ together with the modulus of the std gradient. Within clouds, the variation of std with time can be linked to the particle shift to neighboring Doppler bins due to the presence of micro-turbulence within the same resolution volume. Because of the randomness of the Doppler shifts, the standard deviation of the signal usually decreases as the time averaging increases as shown in Fig. 5b. As previously mentioned, the modulus of the std gradient (i.e. the slope) is also monitored. Low fluctuations of the slope ensure homogeneity of the signal over time. In this work, $N_{\mathrm{av}, \min }$ (corresponding to a minimum averaging time) is set when the std coefficient and the modulus of its gradient become lower than 0.15 and 0.015 respectively, corresponding to the polarimetric accuracy of the TARA radar. $N_{\mathrm{av}, \min }$ is equal to 5 ( $7.5 \mathrm{~s}$ averaging time) in the example displayed in Fig. 5.

\section{Correlation factor $\boldsymbol{R}_{\mathrm{d}}$}

When averaging over a time period longer than $N_{\mathrm{av}, \min }$ (i.e. over a large number of spectra $N_{\mathrm{av}}$ ), multi-modal signals with coherent maxima at specific Doppler bins can occur. Beside, Doppler shifts and spectral broadening of the full Doppler spectra can also appear when averaging over a time period longer than $23 \mathrm{~s} \mathrm{(} N_{\mathrm{av}}>15$ spectra averaged), as shown in Fig. 6. These stationary spectral processes over time are related to real microphysical or dynamical variations within the cloud being probed and can occur in less than a 


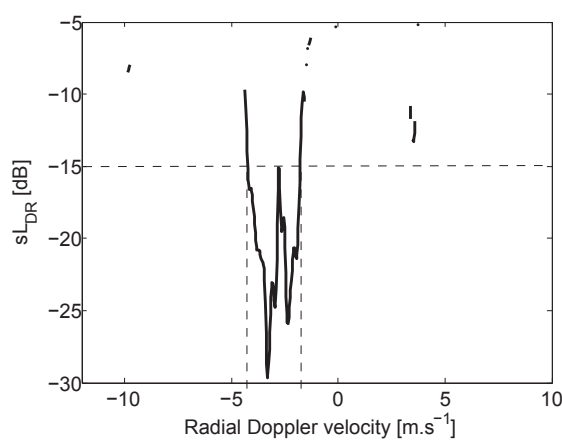

(a)

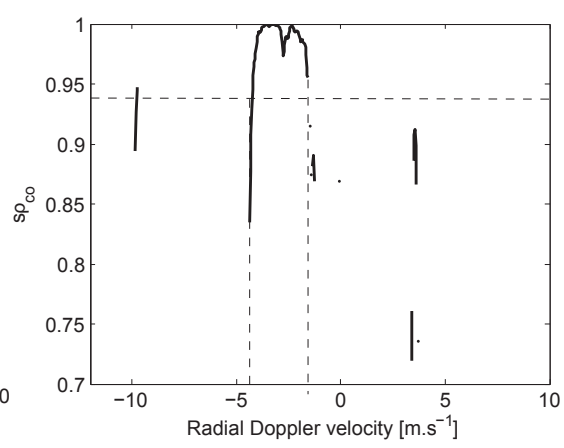

(b)

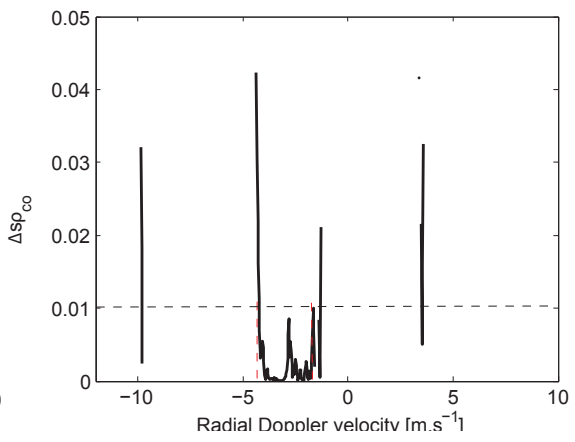

$+$

(c)

Fig. 4. Additional clipping performed on (a) $s L_{D R, V}(v)$ (at $15 \mathrm{~dB}$ ), (b) $s \rho_{\mathbf{c o}}(v)$ (at 0.94) and (c) $\Delta s \rho_{\mathbf{c o}}(v)$ (at 0.1). This additional clipping gives the opportunity to constrain the Doppler velocity window (from $-4 \mathrm{~m} \mathrm{~s}^{-1}$ to $-1.8 \mathrm{~m} \mathrm{~s}^{-1}$ in this example).

(a)
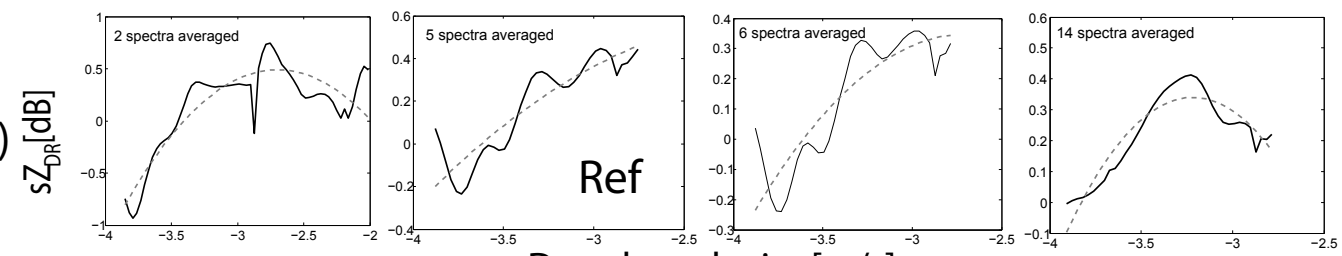

(b)

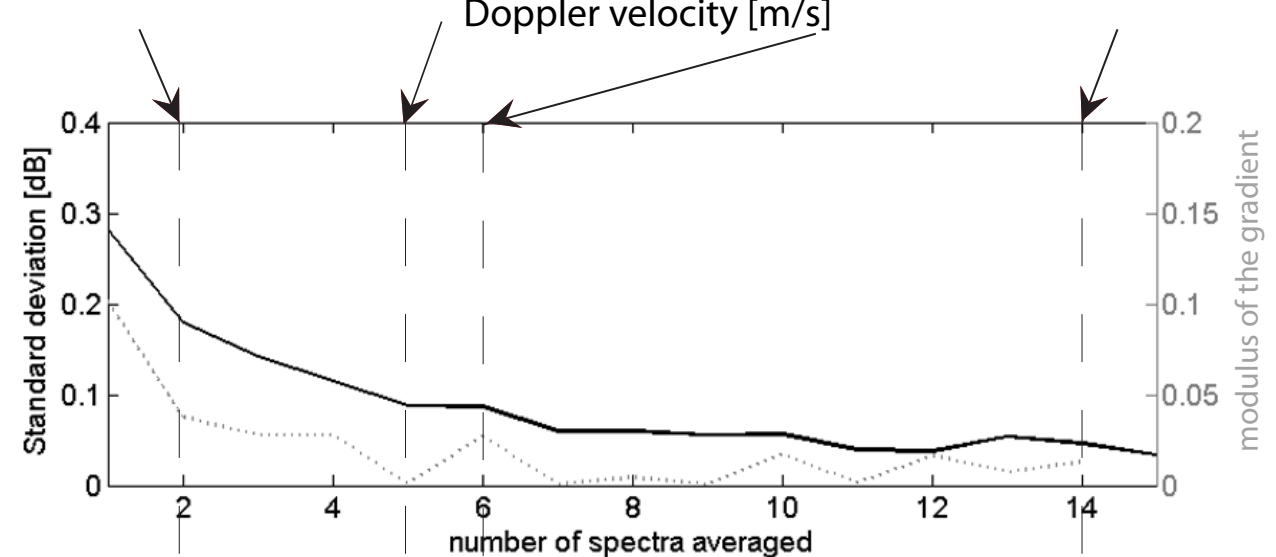

(c)

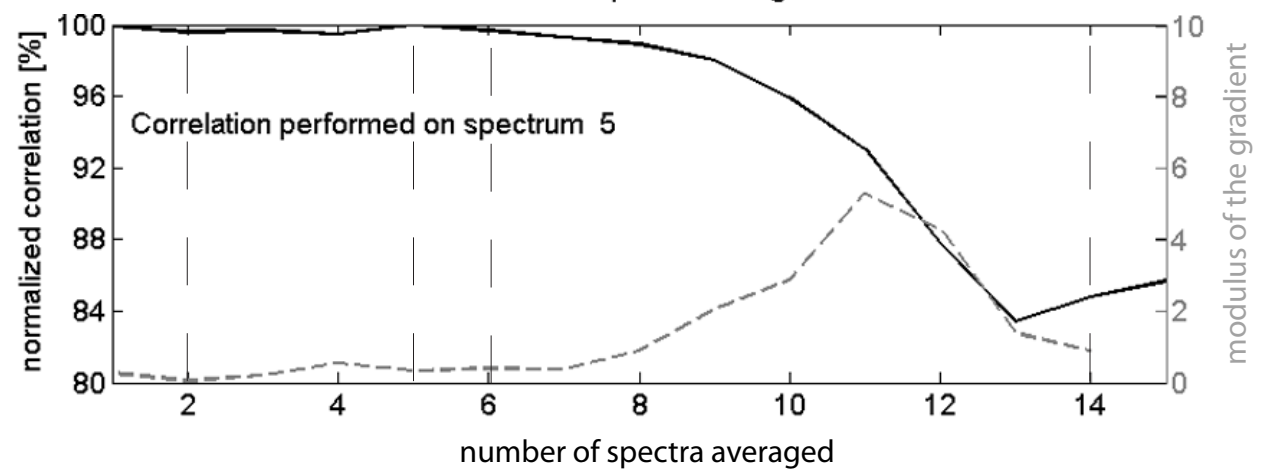

Fig. 5. Example of statistical analysis performed with $s Z_{\boldsymbol{D} R}$ parabolic fit on 15 consecutive spectra for a specific initial time and range bin of a TARA measurement. (a) Representation of the $\boldsymbol{s} \boldsymbol{Z}_{\boldsymbol{D} \boldsymbol{R}}$ spectra obtained with their parabolic fit for different numbers of spectra averaged (i.e. different averaging times). (b) Evolution of the standard deviation coefficient and the modulus of its gradient with the number of spectra averaged. (c) Evolution of the correlation coefficient $R_{\mathrm{d}}$ for $N_{\mathrm{av}, \min }=5$ (average on 5 spectra), where std is found below $0.15 \mathrm{~dB}$. 


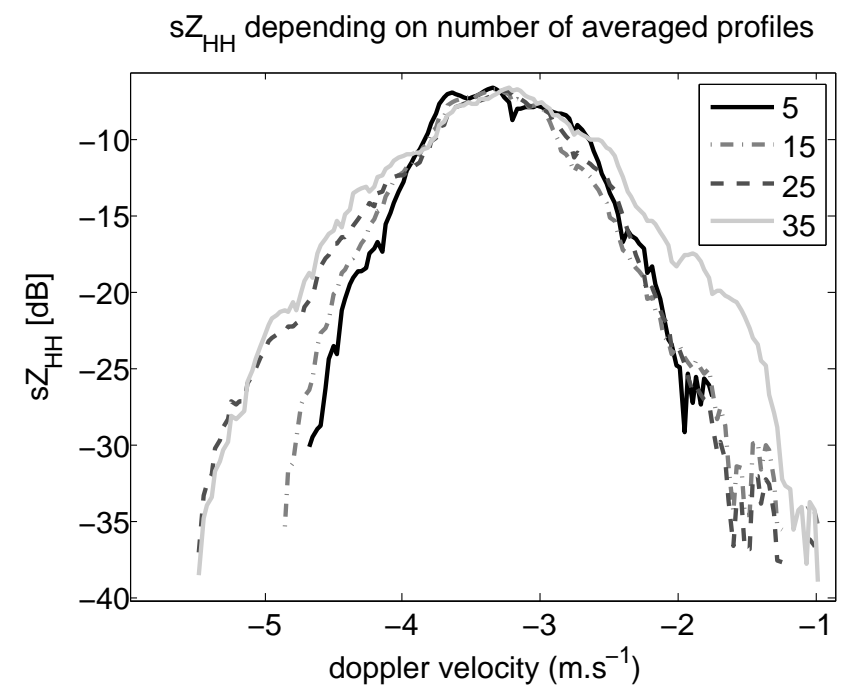

Fig. 6. Broadening effect of $\boldsymbol{s} \boldsymbol{Z}_{\boldsymbol{H} \boldsymbol{H}}(v)$ when an increasing number of spectra is averaged.

few seconds. They do not strongly affect the std analysis but they can drastically change the $\boldsymbol{s} \boldsymbol{Z}_{\boldsymbol{D} \boldsymbol{R}}$ slope, and therefore, the coherency of two consecutive Doppler spectra. The correlation factor $R_{\mathrm{d}}$ is computed in order to monitor the spectral coherency of the parabolic fit over time:

$R_{\mathrm{d}}=\frac{\sum_{v}\left(s Z_{D R, N_{\mathrm{ar}, \text { min }}}^{\mathrm{fit}}(v)-\left\langle s Z_{D R, N_{\mathrm{av}, \text { min }}}^{\mathrm{fit}}\right\rangle\right)\left(s Z_{D R, N_{\mathrm{av}}}^{\mathrm{fit}}(v)-\left\langle s Z_{D R, N_{\mathrm{av}}}^{\mathrm{fit}}\right\rangle\right)}{\sqrt{\sum_{v}\left(s Z_{D R, N_{\mathrm{ar}, \text { min }}}^{\mathrm{fit}}(v)-\left\langle s Z_{D R, N_{\mathrm{av}, \text { min }}}^{\mathrm{fit}}\right\rangle\right)^{2}\left(s Z_{D R, N_{\mathrm{av}}}^{\mathrm{fit}}(v)-\left\langle s Z_{D R, N_{\mathrm{av}}}^{\mathrm{fit}}\right\rangle\right)^{2}}}$.

In Eq. (7), $N_{\mathrm{av}, \min }$ is fixed and $N_{\mathrm{av}}$ varied from 1 to 15 . The spectra averaged over $N_{\mathrm{av} \text {,min }}$ is taken as reference. $R_{\mathrm{d}}$ is expressed in percent, and is equal to $100 \%$ for two identical spectra, i.e. $100 \%$ correlation. Figure $5 \mathrm{c}$ displays the evolution of $R_{\mathrm{d}}$ as well as the modulus of its gradient. For a reliable microphysical retrieval, high coherency within nearby averaged spectra should be observed. This guarantees that measurements are performed within a homogeneous resolution volume in terms of microphysical and meso-scale dynamical cloud properties. In previous work, Dufournet (2010), showed that more than $10 \%$ decorrelation of the $s Z_{\boldsymbol{D} R}$ signal could strongly affect the output of the microphysical retrieval technique, and mainly the concentration of oriented ice particles. A $10 \%$ threshold is therefore adopted within this paper. Averaged spectra showing more than $10 \%$ decorrelation are discarded. In the example taken in Fig. $5 \mathrm{c}$, all spectra averaged with $N_{\text {av }}$ greater than 12 (averaging time above $18 \mathrm{~s}$ ) are not considered coherent and cannot be averaged.

At this stage of the processing, a final averaging time (or number $N_{\text {av }}$ spectra to average) can be obtained. It corresponds to the minimum averaging time for which all the three thresholds mentioned above (i.e. on std and the modulus of its gradient, and $R_{\mathrm{d}}$ ) are satisfied. In Fig. 5, for example, $N_{\mathrm{av}}=5$, corresponding to an averaging time of $7.5 \mathrm{~s}$, is chosen in order to remove any microphysical and dynamical fluctuations in the averaged Doppler spectrum.

An example is given in Fig. 7 for consecutive spectra obtained, before (middle row) and after (bottom row) averaging over a specific time amount (specific $N_{\mathrm{av}}$ value) determined from the statistical analysis. Each spectrum from left to right is obtained within an ice cloud event at $1.5 \mathrm{~s}$ interval.

\section{Application for ice cloud microphysical retrievals}

Compared to other spectral polarimetric parameters, $s \boldsymbol{Z}_{\boldsymbol{D} \boldsymbol{R}}(v)$ can provide straightforward information on particle orientation dominating the signal for a specific Doppler interval (positive when horizontally aligned particles dominate the signal, negative when vertically aligned and close to zero when particles do not exhibit any specific orientation). $\quad \boldsymbol{s} \boldsymbol{Z}_{\boldsymbol{D} \boldsymbol{R}}(v)$ is therefore chosen in this paper to introduce the potentiality of spectral polarimetry for microphysical retrieval purposes as well as to categorize the microphysical diversity within the medium being probed. In this section, particle habit(s) and orientation(s) are retrieved from TARA observations within ice/mixed-phase clouds. It is important to note that this method is still in a preliminary stage and is therefore only described in order to illustrate the potential of using spectral polarimetric parameters in microphysical retrievals. At the TARA working frequency of $3.3 \mathrm{GHz}$, only cloud ice crystals and falling drops (drizzle and rainfall) are detected within the measurements, cloud water and supercooled water droplets being too small (mean diameter smaller than $20 \mu \mathrm{m}$ ) to be discerned by the radar. If we assume that most ice crystals grow rapidly to a recognizable non-spherical shape larger than $25 \mu \mathrm{m}$ due to high supersaturation with respect to ice (Lawson et al., 2001), TARA can be used to provide direct ice crystal observations without being affected by the supercooled water phase in mixed-phase cloud regions.

The microphysical retrieval algorithm investigated here is based on different spectral and integrated polarimetric parameters, that is, $s Z_{D R}(v), s \rho_{\mathbf{c o}}(v)$ and $L_{D R}$. The retrieval follows the steps below.

\subsection{Partitioning of cloud particle type}

As previously mentioned, most radar resolution volumes can contain different hydrometeor types which evolve within the same cloud volume. The probability of particle mixing within the radar resolution volume is even more important at far distances from the radar as the radar volume expands with distance. Most current retrieval algorithms assume the microphysical homogeneity of the medium being retrieved. Thus, cloud particle mixing can lead to wrong characterization of parameters, including particle number densities, shapes and phases which, in turn, affect the microphysical retrieval outputs. 


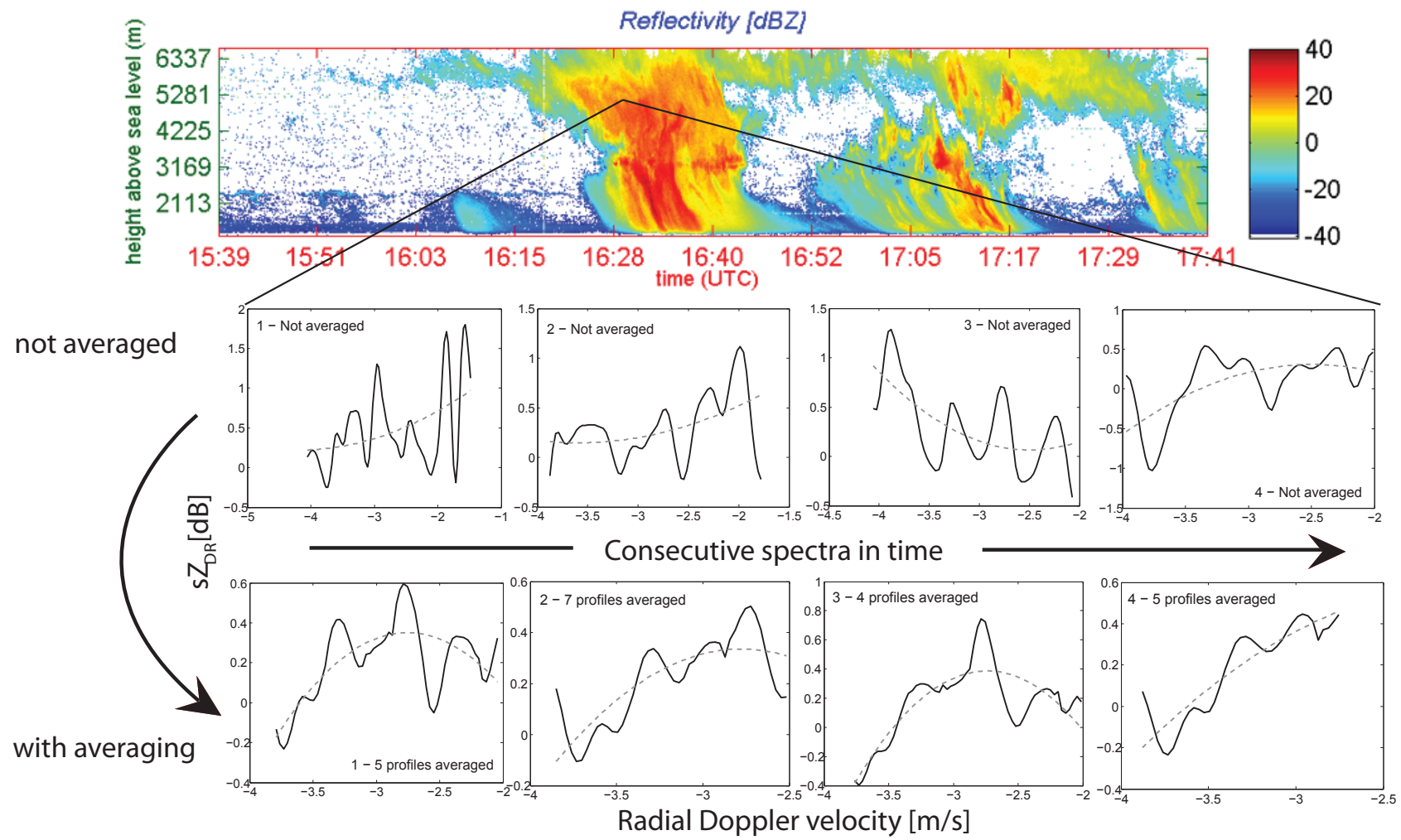

Fig. 7. Four consecutive $s Z_{D R}$ spectra extracted from an ice cloud region (see reflectivity profile). Each spectrum is measured at $1.5 \mathrm{~s}$ interval and averaged (bottom row) over a different averaging time. The set of spectra are represented without (middle row) and with (bottom row) the additional averaging step. As displayed, the spectra become more coherent when the averaging is performed.

Hydrometeors detected in each radar volume are spectrally classified into different groups based on the similarity of their sizes, shapes and main orientations. Because $s Z_{D R}(v)$ is related to such microphysical parameters (see Sect. 2.1), the algorithm uses variations detected in the slope of $s \boldsymbol{Z}_{\boldsymbol{D} \boldsymbol{R}}(v)$ over the whole spectrum in order to infer changes in particles properties. Parabolic fit on $\boldsymbol{s} \boldsymbol{Z}_{\boldsymbol{D R}}(v)$ is used for particle classification in order to discard higher order fluctuations of the original $s \boldsymbol{Z}_{\boldsymbol{D} \boldsymbol{R}}$ spectrum which would affect the classification. Within a set of cloud observations performed with TARA, 16 main particle arrangements, each comprising 1 to 3 groups of particles, have been extracted from the parabolic fit made on the slope of the $\boldsymbol{s} \boldsymbol{Z}_{\boldsymbol{D} \boldsymbol{R}}$ spectrum, as displayed in Fig. 8. A categorization number $N_{\text {cat }}$ is attributed to each radar volume for which the retrieval is executed. For the sake of clarity, a schematic representation of ice crystal's orientation and habit is added on the left hand side of Fig. 8 .

\subsection{Characterization of ice crystal orientation}

Ice crystal orientation is caused by the airflow passing around the particle. This airflow creates low pressure at the crystal edges resulting in a torque which tends to align the axis of symmetry of the particle along the airflow's direction (Brussaard, 1976). However, different dynamical mechanisms, such as windshear or strong updraft, can act upon the airflow's orientation, changing the particle's orientation. Crystal orientations play a role in estimating particle fall velocities (Mitchell et al., 1996), which are often used for the determination of the particle size distribution. A good estimation of ice crystal's orientation is therefore required.

At the TARA wavelength $(\approx 10 \mathrm{~cm})$, ice particle orientation can be directly obtained from the mean $s Z_{D R}$ value(s) measured for each group of particles detected in the same resolution volume. A fairly simple orientation algorithm is currently implemented in the microphysical retrieval. Each ice crystal species is assumed to be composed of either horizontally, vertically or randomly oriented particles. For a Doppler spectrum interval (related to a specific group of ice crystals) with positive $\boldsymbol{s} \boldsymbol{Z}_{\boldsymbol{D R}}(v)$, the predominant ice particles are assumed to be horizontally aligned. This is the case for some ice crystal groups detected in radar volumes with $N_{\text {cat }}=10$ or above. Conversely, negative $s \boldsymbol{Z}_{\boldsymbol{D} \boldsymbol{R}}(v)$ are related predominantly to vertically oriented ice crystals $\left(0<N_{\text {cat }}<9\right)$. Ice crystals present in Doppler intervals where $s Z_{D R}(v)$ is close to zero is quite ambiguous. On the one hand, $\boldsymbol{s}_{\boldsymbol{D} \boldsymbol{R}}(v)=0$ can be interpreted as particles having spherical or close to spherical shapes producing equal radar cross-sections in horizontal and vertical polarization state. On the other hand, 


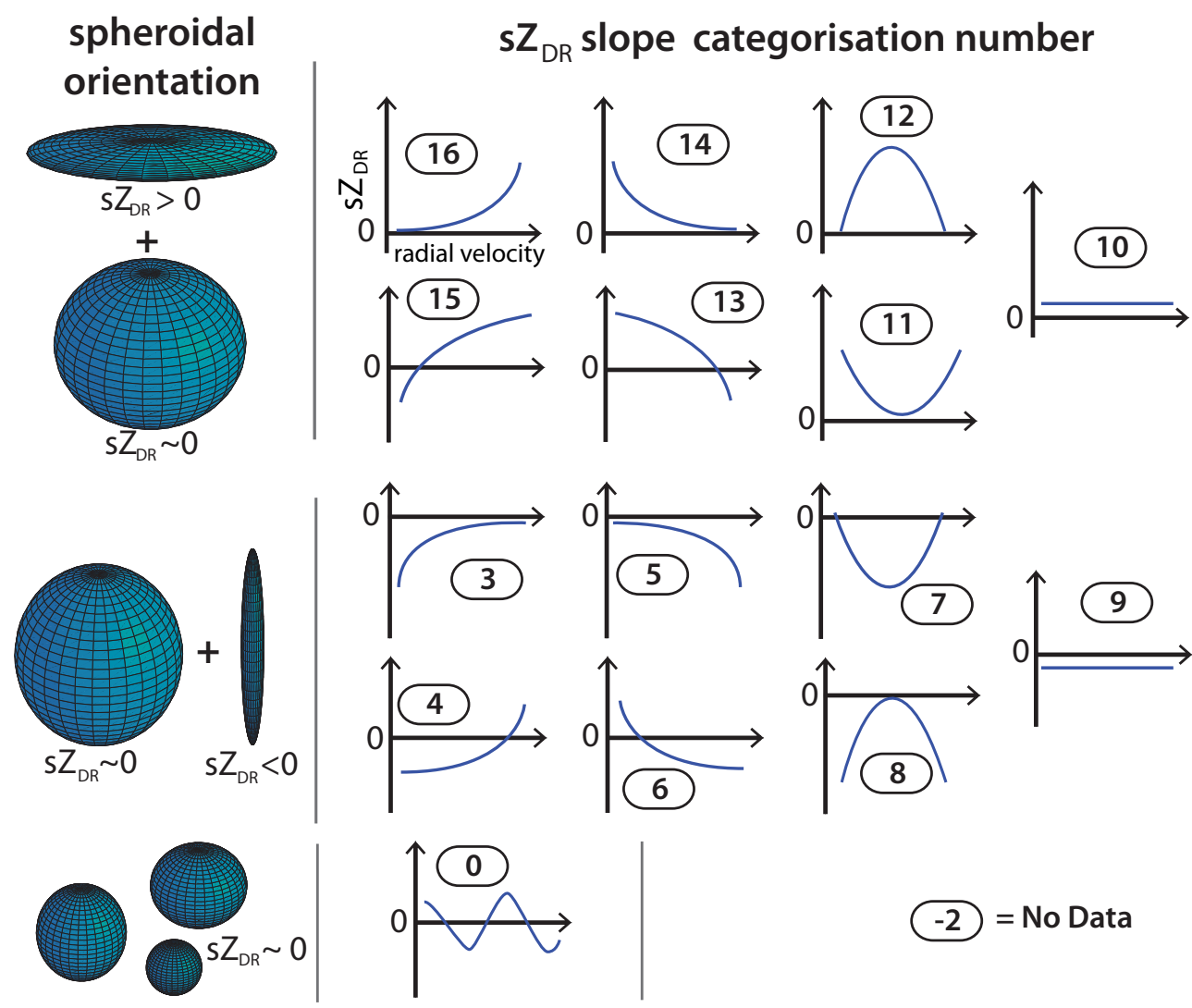

Fig. 8. $s Z_{D R}$ classification based on the slope and values of the spectra. 16 different $s Z_{D R}$ configurations are sorted out from cloud observations. On the left hand side, a spheroidal representation of the ice crystals shape(s) and orientation(s) contained in the same resolution volume is displayed for illustration, depending on the Doppler spectral configuration.

turbulence effects can also cause random particle orientation with $s \boldsymbol{Z}_{\boldsymbol{D} \boldsymbol{R}}(v)$ fluctuating around zero across the spectrum. In this paper, it is assumed that aggregates, graupel and hail can be assimilated to near spherical particles $\left(s \boldsymbol{Z}_{\boldsymbol{D} \boldsymbol{R}}(v)\right.$ around zero) as aggregation and riming processes reduce primary habits of the newly formed ice crystal. Due to their large sizes, such particles are also less mean ambient wind dependent than pristine ice, making them more randomly aligned.

\subsection{Characterization of ice crystal habit:}

More than 60 ice crystal species have been observed within natural clouds (Mogano and Lee, 1966). However, this classification can be simplified by merging particle habits into three categories while still keeping a fairly good approximation of the particle shape: pristine ice crystals (comprising plate, dendrite, column and needle-like ice crystals), aggregates, heavily rimed crystals (graupel and hail). As for the orientation, particle habits also affect most of the microphysical retrievals, a wrong shape assumption leading to wrong particle density, wrong axis ratio (if ice crystal shapes is assumed spheroidal), and wrong fall velocity computations.
The particle habit retrieval technique presented is meant to work within mixed-phase cloud having low riming processes (graupel and hail are not taken into account). Under such a condition, a simple categorization scheme can be employed, partitioning particle into the three following groups: aggregates, plate-like crystals and column-like crystals. Again, different spectral polarimetric tools are used for the shape/habit categorization. The spectral cross-correlation parameter is first investigated in order to distinguish the aggregates from the pristine ice crystals. Figure 9 represents a typical distribution of $\boldsymbol{s} \rho_{\text {co }}$ values over the whole Doppler spectrum that one can expect for two different microphysical situations. On the left side, a combination of plates and aggregates was observed from collocated aircraft measurements while only aggregates was present within the right hand side distribution. High $\boldsymbol{s} \rho_{\text {co }}$ values, above 0.995 , are found when aggregates predominate in the full radar resolution volume (i.e. as assumed in this paper for Doppler intervals where $\boldsymbol{s} \boldsymbol{Z}_{\boldsymbol{D} \boldsymbol{R}}(v)$ close to zero). Conversely, smaller $\rho_{\text {co }}$ values $\left(0.95<s \rho_{\text {co }}(v)<0.97\right)$ can be observed and also simulated from different pristine ice crystals when the radar antennas are tilted at $45^{\circ}$ elevation angle. Because mixing different particle types affects $s \rho_{\mathbf{c o}}(v)$, a weighting factor 


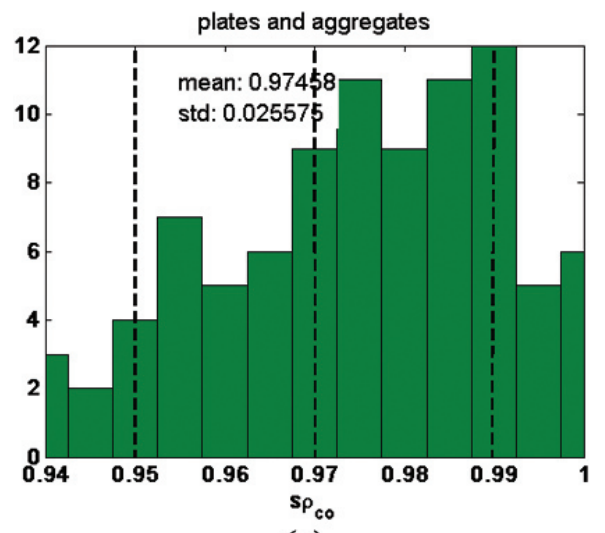

(a)

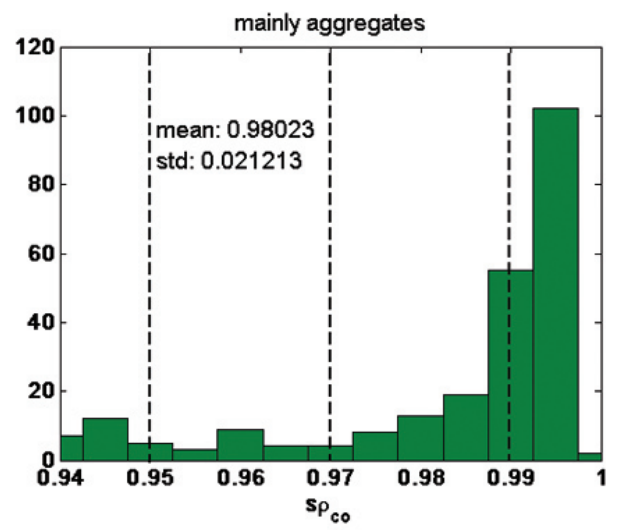

(b)

Fig. 9. Typical histograms obtained from spectral cross-correlation coefficients derived from TARA (a) when pristine crystals (plates in that case) and aggregates are observed (aircraft observations) in the radar resolution volume and (b) when mostly aggregates are observed in the radar resolution volume.

$R_{\mathrm{W}}$ is created for the particle habit categorization and defined so that

$$
R_{\mathrm{W}}=\frac{\sum_{i=0.995}^{i=1} N_{i}}{\sum_{i=0.95}^{i=0.97} N_{i}},
$$

where $N_{i}$ is the total number of spectral samples per $\boldsymbol{s} \rho_{\text {co }}$ intervals $i$. This $R_{\mathrm{W}}$ ratio is preferred rather than using the mean or the standard deviation of the distribution, as such parameters can give similar values for different microphysical situations (see mean and std of the distribution difference in Fig. 9a-b). When $R_{\mathrm{W}}<2$ (threshold determined empirically), pristine ice crystals dominate the radar resolution volume. Aggregates dominate in the other cases with the level of aggregation increasing with $R_{\mathrm{w}}$.

Secondly, the type of pristine ice crystals (plate- or column-like ice crystals) was investigated from the combination of $R_{\mathrm{W}}$ with the linear depolarization ratio $L_{D R}$. As mentioned in Sect. 2.1, $L_{D R}$ strongly depends on the axis ratio and canting angle of the particles. $L_{D R}$ increases when increasing the standard deviation of the distribution of canting angle of all hydrometeors, i.e. passing from perfectly to randomly oriented ice crystals. As a result, significant differences in $L_{D R}$ between plate- and column-like crystals are expected when a non-random orientation is considered, according to Battaglia et al. (2001). For the categorization algorithm, a first threshold guess was employed to distinguish plates from columns within pristine ice regions. At 45 degrees elevation angle, simulated $L_{D R}$ distributions centered around $-15 \mathrm{~dB}$ suggested the presence of columns whereas $L_{D R}$ is mostly below $-20 \mathrm{~dB}$ for plate-like ice crystals. A summary of the crystal habit categorization is provided in Table 2.

\subsection{Case study of 21 July 2007}

During summer 2007, the TARA radar took part in the Convective and Orographically-induced Precipitation Study (COPS) with the aim of studying heavy precipitation events driven by orographic conditions at high resolution scales. Precipitation processes were observed by means of a synergetic collection of research remote-sensing and/or in-situ systems operated at the ground, on-board aircraft and satellite. Six main ground-based sites were deployed across the COPS domain. TARA was located on the Hornisgrinde site $(\mathrm{H})$, on top of a plateau, close to other ground-based sensors. More information on the campaign can be found in Wulfmeyer et al. (2008). In this paper, Saturday, 21 July 2007, is taken as a case study period in order to illustrate the microphysical approach described in the previous sections.

During that day, a frontal zone was affecting the COPS area with cold air in the north and warm air in the south. A wave (with presence of a high pressure ridge) was developing and stretching southwest to northeast up to the COPS domain, leading to the formation of a low pressure area over Bavaria. This system was responsible for upward vertical motion and the formation of a Mesoscale Convective System (MCS) moving northeastwards over the eastern half of the COPS area in the course of the afternoon. In terms of cloud coverage, widespread clouds, turning to convective (enhanced by the orography) and rainy cells were observed in the afternoon. TARA was operated to sample the MCS over the COPS domain. The TARA measurements were combined with synchronous aircraft in-situ measurements performed with the ATR42 aircraft from Safire, in the framework of the Observation Strategy for Mixed-phase Orographic Clouds project (OSMOC) under the umbrella of the European Fleet for Airborne Research activities (EUFAR). 
Table 2. Crystal habit categorization.

\begin{tabular}{ll}
\hline Ice particle type present in the resolution volume & Categorization parameters \\
\hline aggregates dominating the Doppler spectra & $N_{\mathrm{cat}}=0$ and $R_{\mathrm{W}}>>2$ \\
Column-like crystals dominating the Doppler spectra & $R_{\mathrm{W}}<2$ and $L_{D R} \approx-15 \mathrm{~dB}$ \\
Plate-like crystals dominating the Doppler spectra & $R_{\mathrm{W}}<2$ and $L_{D R} \approx-20 \mathrm{~dB}$ \\
\hline
\end{tabular}
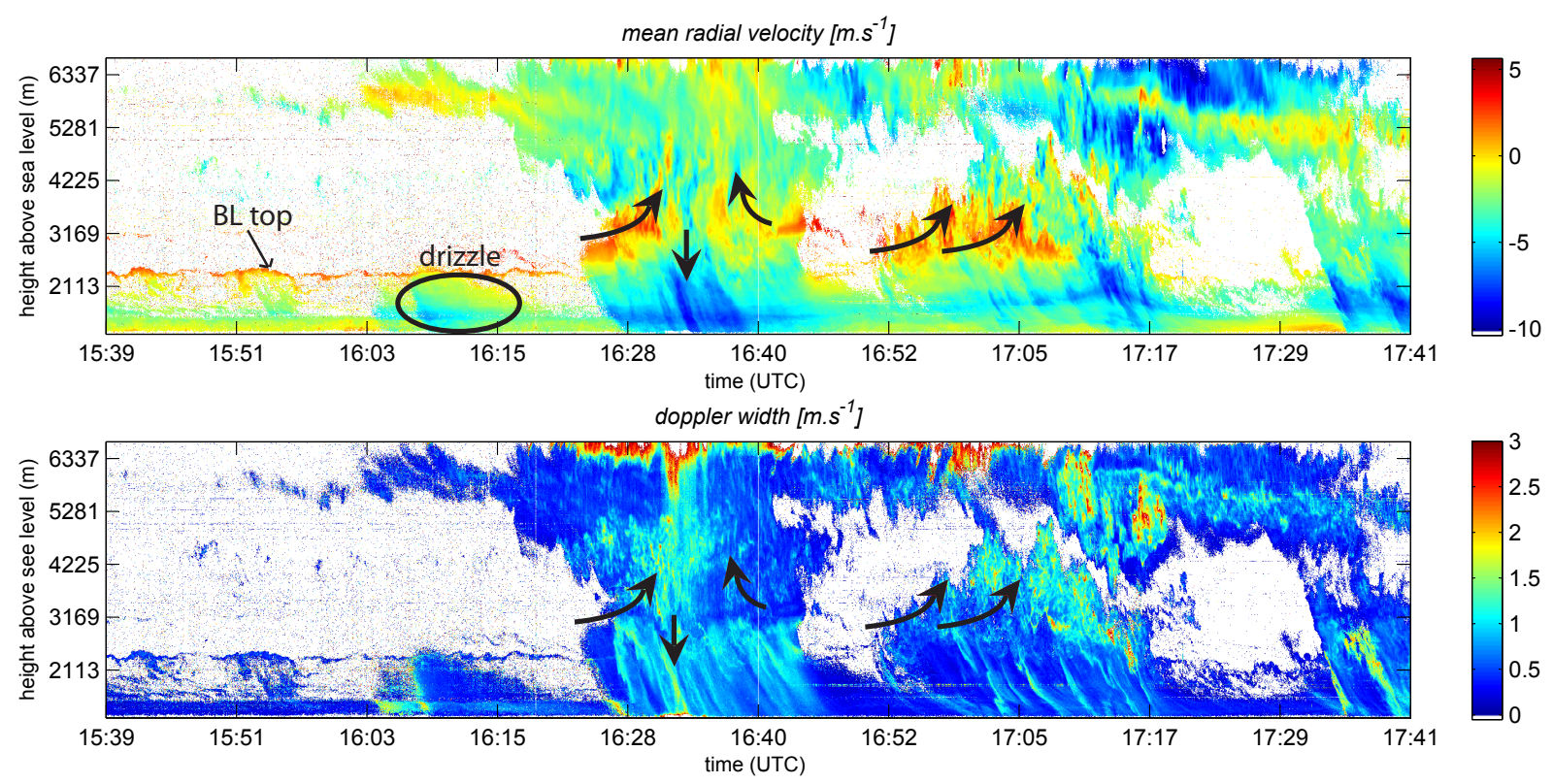

Fig. 10. Radial Mean Doppler velocity (top) and Doppler width (bottom) measured by the TARA radar on 21 July 2007.

\subsubsection{TARA measurements and interpretation}

TARA started observations at 13:05 UTC at 45 degrees elevation angle. Figure 10 represents the radial mean Doppler velocity profiles and the Doppler width profiles as measured by the TARA radar from 15:39 UTC onwards. The sign convention of the radial mean velocity is taken such that values are positive when particles move away from the radar and negative otherwise. On 21 July 2007, rapid scan images obtained with Meteosat Second Generation could be used to estimate the main wind direction, which was found orthogonal to the line of sight of the radar. For this direction, the horizontal wind component of the radial mean velocity is considerably weakened. Also considering that, in the first $4000 \mathrm{~m}$, low horizontal radial wind velocities of less than $10 \mathrm{~m} \mathrm{~s}^{-1}$ were measured by a radiosonde launch on the same site, the mean vertical wind component is most probably dominating in the radial mean velocity measurements, at the expense of the horizontal wind component. Therefore, the radial mean velocity profile can be used to qualitatively characterize the vertical wind evolution. On 21 July 2007, analyses of the mean Doppler velocity and Doppler width profiles revealed the presence of a strong updraft (Dufournet, 2010) proba- bly enhanced by orography acting on a westerly wind flow, which could play a role on particle orientation.

Figure 11a shows the reflectivity profiles obtained from 15:45 UTC onwards during that day. The MCS captured from 16:20 UTC to 16:45 UTC was taken as case study for the microphysical retrieval. During this time slot, a cloud with great vertical extension from $3200 \mathrm{~m}$ to $6400 \mathrm{~m}$ (nimbostratus type) was observed. Because of the high reflectivity encountered in this mid-level cloud region (above 10 dBZ), ice crystals were very likely present. Just below $3200 \mathrm{~m}$, a bright band was detected indicating the melting of the precipitating ice crystals. Quite heavy precipitation resulted from the melting process, with reflectivity values reaching $40 \mathrm{dBZ}$. From a dynamical point of view, analyses of the mean Doppler velocity and Doppler width profiles revealed the presence of a strong updraft (Dufournet, 2010) probably enhanced by orography acting on a westerly wind flow, which could play a role on particle orientation. 


\subsubsection{Microphysical retrieval results}

The microphysical retrieval technique was applied to the ice/mixed-phase cloud passing over the $\mathrm{H}$ site from 16:20 UTC to 16:45 UTC, where high signal to noise ratio was detected. The black box in Fig. 11a bounds the retrieval region and a zoom of the reflectivity profile within the same box is provided in the same image. Retrievals are displayed in Figs. $11 \mathrm{~b}$ to e. The mapping of particle type and orientation categorization procedures, explained in Sects. 3.1 and 3.2, is represented in Fig. 11b using a simplified color scale. Some of the categorization numbers $N_{\text {cat }}$ defined in Fig. 8 are merged with respect to the particle orientation(s) and number of species present within each radar resolution volume. On the one hand, light yellow pixels, grouping $N_{\text {cat }}=12,14$ or 16 (radar volume containing up to two cloud particle types) and $N_{\text {cat }}=11,13$ or 15 (radar volume containing at least two cloud particle types), represent cloud volumes where at least one particle type is horizontally aligned. On the other hand, $N_{\text {cat }}=3,5$ or 7 and $N_{\text {cat }}=4,6$ or 8 represent vertically aligned ice particles. Aggregates are present within each radar volumes. However, a specific color scale for $N_{\text {cat }}=0$ is defined when it is assumed that only randomly oriented aggregates fill the resolution volume. Oriented particles were often detected in this cloud. A change of orientation with time was also observed around 16:30 UTC. This shift of orientation was closely linked to a change in the Doppler width coefficient, which could arise from a strong variation of the amplitude of the horizontal wind, leading to production of turbulence and changes in ice crystal orientation.

Looking at the relative proportion of oriented and randomly oriented particles, it was noticed that oriented particles (mainly horizontal alignment) were much more represented at cloud top. This trend was reversed as reaching cloud bottom as seen in Fig. 11b. Figure 11c maps the weighting coefficient defined in Sect. 3.3. Because $R_{\mathrm{w}}$ was low at cloud top, oriented particles found in this region were pristine ice particles. Combining this information with the mean of the $L_{D R}$ distribution found for the same cloud region $\left(L_{D R} \approx-21 \mathrm{~dB}\right.$ as seen in Fig. $\left.11 \mathrm{~d}\right)$, horizontally oriented plate-like particles were most probably located at cloud top. As we go farther down, $R_{\mathrm{w}}$ increased, suggesting the occurrence of ice crystal aggregation processes. Even some strong aggregation areas at cloud bottom, with $R_{\mathrm{W}}>4$, were observed. This result is shown in the simplified categorization of Fig. 11e. From this figure, good physical consistency was found between the cloud processes and the precipitation regimes observed below the melting layer, e.g. strong aggregation areas connected to heavy rainfall regimes as explained in Dufournet (2010).

\subsubsection{Ice crystal habit assessment using the COPS facilities}

An ATR42 flight was coordinated with the TARA measurements on 21 July 2007, in the frame of the OSMOC project, taking advantage of the COPS facilities, i.e. a specific daily weather forecast dedicated to the COPS area coupled with a dense measurement network providing a detailed nowcasting of the cloud overcast. This ATR42-320 aircraft is modified for scientific use, and has flying performances suitable for the study of ice/mixed-phase clouds in complex terrain. In order to satisfy the needs of the OSMOC experiment, a sensor configuration focused on microphysical measurements was adopted. For particle habit assessment, the 2D$\mathrm{C}$ probe images obtained during in-cloud penetrations were analyzed at different flight levels (Fig. 12a). Plate and dendrites, mixed with small aggregate particles, were mainly found at cloud top (about $5000 \mathrm{~m}$ altitude). For flight legs performed at lower altitude, only aggregates at different riming stages were observed. This result is consistent with the retrieved ice particle habits obtained from TARA spectral polarimetric parameters. Graupel was also observed within a high riming region, but their retrieval is not yet implemented in the algorithm.

Finally, plate-like pristine ice at cloud top is consistent with radiosonde data launched from a site situated $10 \mathrm{~km}$ away from the TARA radar. From Fig. 12b, cloud temperatures below $-10^{\circ} \mathrm{C}$ were found at cloud top, which is favorable for plate-like crystal formation according to Lamb and Scott (1972).

\section{Conclusions}

Most cloud microphysical retrieval techniques suffer from a lack of microphysical information provided by current observations, such as radar sensors, for the microphysical characterization of ice/mixed-phase clouds. Several assumptions are required to fill in this lack of characterization, which strongly affects the retrieval accuracy needed for a correct and complete cloud microphysical description, including particle number densities, orientations and types within the same cloud volume (Donovan et al., 2005; Matrosov et al., 2000). In this paper, we have investigated the use of simultaneous Doppler and polarimetric radar measurements for microphysical retrievals within ice/mixed-phase clouds. First results suggested that some relations might exist between spectral polarimetric parameters and the cloud microphysical properties, providing a wealth of additional microphysical information from a single radar signal that could potentially reduce the cloud microphysical retrieval uncertainties.

Several spectral polarimetric parameters obtained from TARA radar data have been analyzed. Because spectral polarimetric parameters are by nature extremely noisy, this analysis has mainly concentrated on how to process them. 


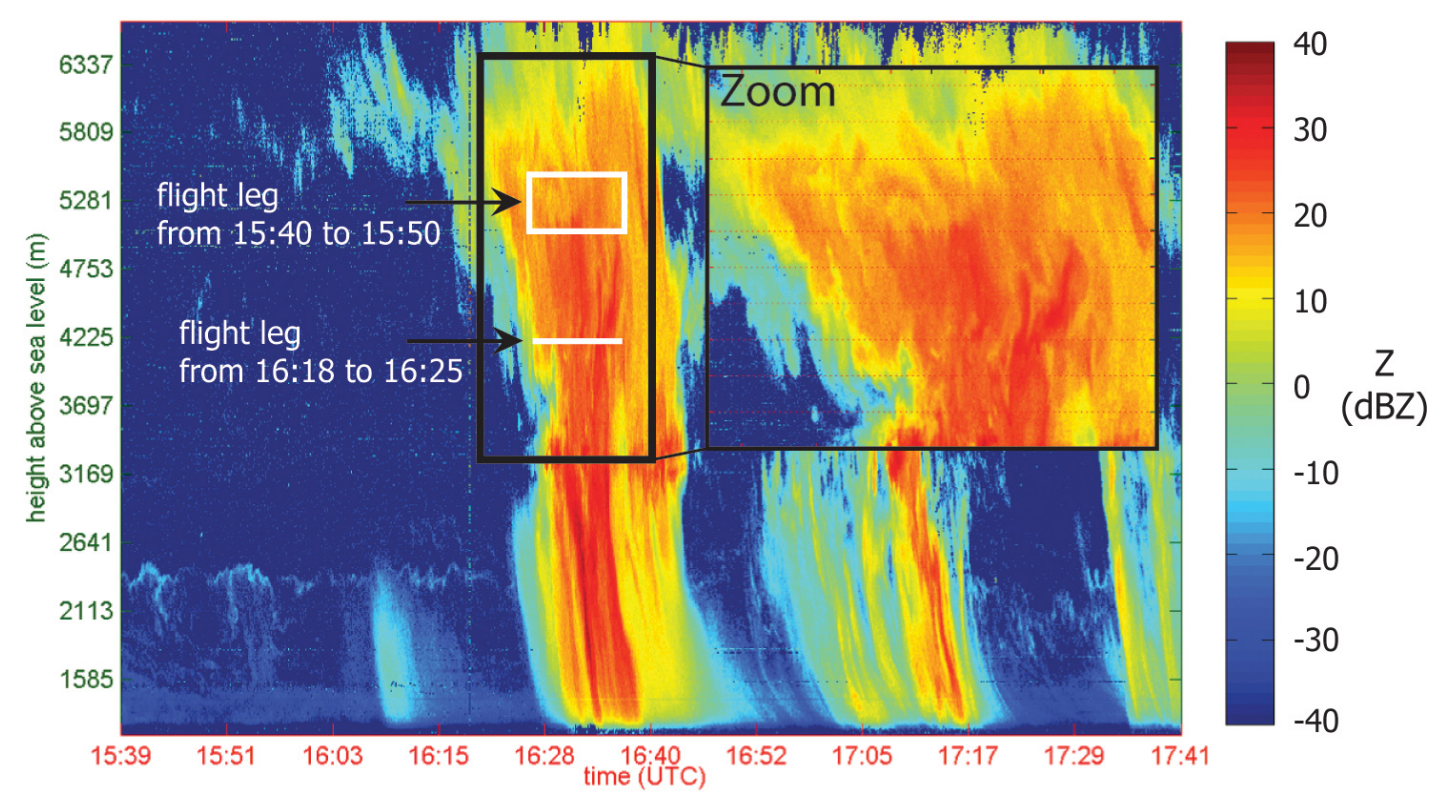

(a)

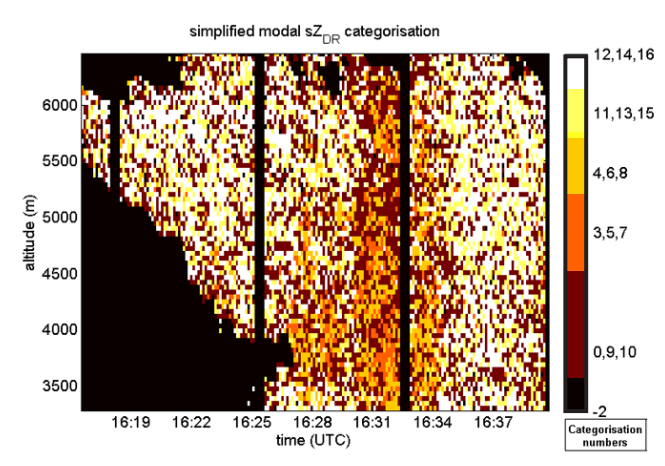

(b)

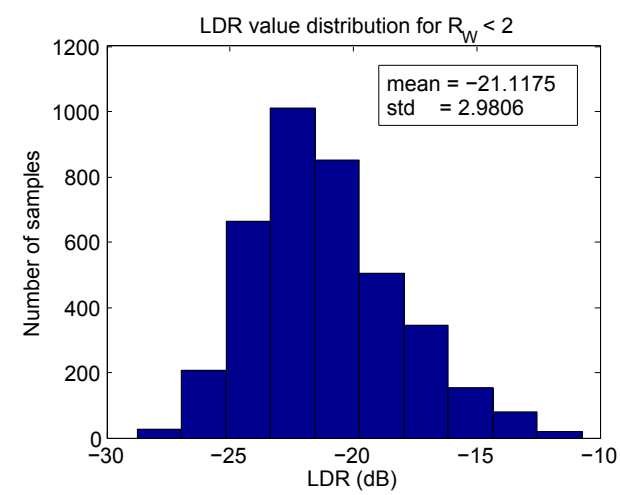

(d)

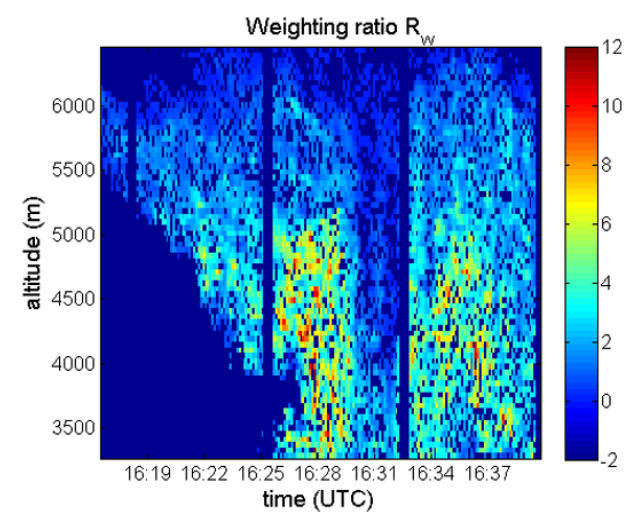

(c)

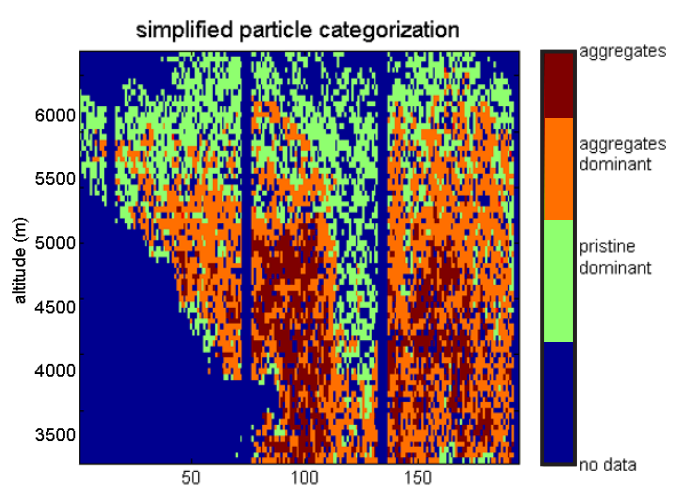

(e)

Fig. 11. Observations and retrievals obtained from the TARA measurements on 21 July 2007. (a) Reflectivity profile from TARA. The black box delimits the boundaries of the region where the retrieval technique is applied. The white box and white line represent the altitude of the flight legs performed by the ATR42 aircraft the same day. (b) Mapping of the $\boldsymbol{s} \boldsymbol{Z}_{\boldsymbol{D} \boldsymbol{R}}$ categorization results. (c) Mapping of the weighting ratio $R_{\mathrm{W}}$. (d) Histogram of $L_{D R}$ values when $R_{\mathrm{W}}<2$. (e) Simplified particle type categorization obtained from (b), (c) and (d). 


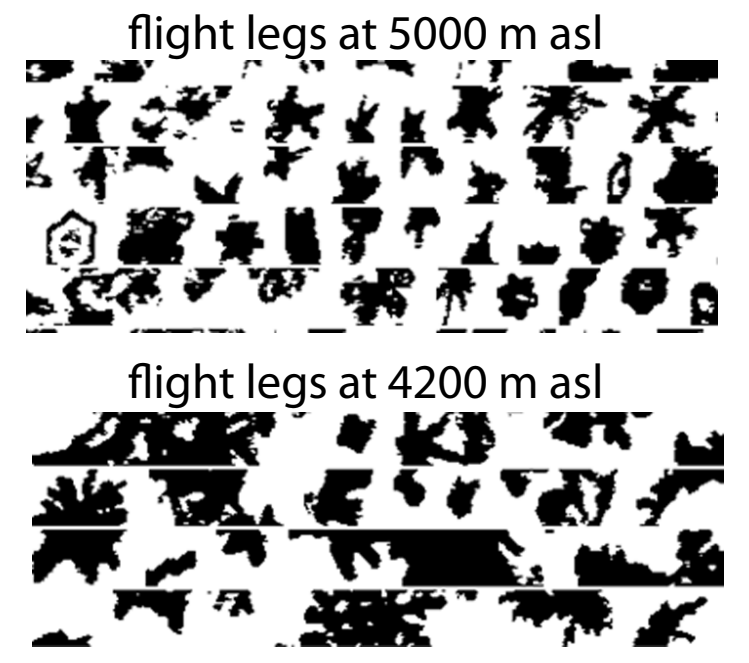

(a)

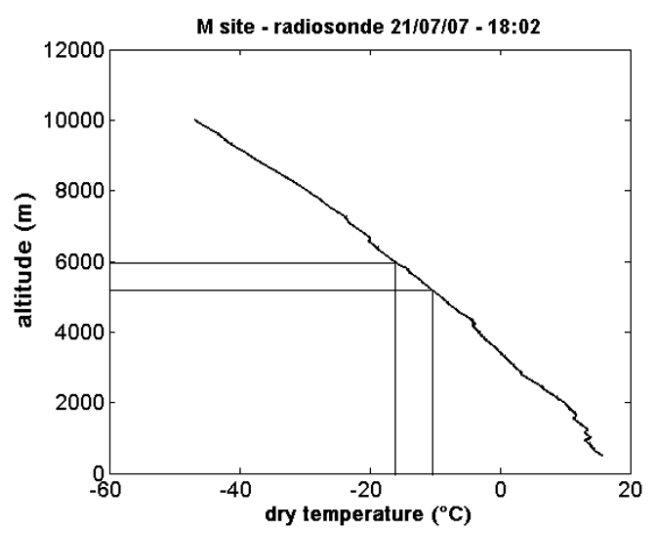

(b)

Fig. 12. (a) 2D-C probe images obtained from the ATR42 aircraft flying through the MCS event on 21 July 2007 at the two different flight levels shown in Fig. 11a. (b) Temperature profile obtained from a radiosonde launched at the M site also on the same day.

This work argues that appropriate signal filtering and adequate time averaging are of great importance to ensure the spatial and temporal coherency of the spectral polarimetric parameters, and thereby, consistent spatial and temporal microphysical retrieval outputs.

In the second half of the paper, simple microphysical retrieval algorithms for number of ice crystal species, main particle orientations and habits were defined and applied to TARA measurements carried out during the COPS campaign on 21 July 2007. The retrieval outputs were found to be consistent with collocated measurements performed on site or on-board the ATR42 aircraft on the very same day, which is encouraging for further investigations. Despite its preliminary character, the research reported here would seem to indicate that spectral polarimetry can provide additional microphysical information not often taken into account in current retrievals, i.e. the number of ice crystal species present in each cloud resolution volume and their main orientation.

The use of retrievals based on spectral polarimetric parameters is likely to be a fruitful area of further research. On the one hand, the retrievals defined in Sect. 3 can be improved for operational use. Also new retrieval algorithms based on spectral polarimetric parameters are currently being investigated for the characterization of particle size distributions within ice/mixed-phase clouds. On the other hand, the algorithms presented here could be implemented within current retrieval algorithms in order to reduce the number of assumptions used. For example, the ice crystal number density, considered to be critical in radar-lidar methods (Delanoe et al., 2006), could better be characterized from the particle habits retrieved from spectral polarimetric measurements.
Acknowledgements. The authors wish to thank the crew of the ATR42 aircraft, the EUFAR organization to make the collection of in-situ data possible and the LAMP institute (Laboratoire de meteorology Physique - Clermont-Ferrand, France) which provided the processed in-situ data. The authors also wish to thank the COPS organization committee for their help in achieving the collocated measurements during the campaign.

Edited by: A. Apituley

\section{References}

Battaglia, A., Sturniolo, O., and Prodi, F.: Analysis of polarization radar returns from ice clouds, Atmos. Res., 59-60, 231-250, 2001.

Bringi, V. and Chandrasekar, V.: Polarimetric Doppler Weather Radar - Principle and applications, Cambridge University Press, 2001.

Brussaard, G.: A meteorological model for rain induced crosspolarization, I.E.E.E. T. AP, USA, 24, 5-11, 1976.

Cantrell, W. and Heymsfield, A.: Production of ice in tropospheric clouds - A review, B. Am. Meteorol. Soc., 86, 795-807, 2005.

Delanoë, J. and Hogan, R. J.: A variational scheme for retrieving ice cloud properties from combined radar, lidar, and infrared radiometer, J. Geophys. Res., 113, D07204, doi:10.1029/2007JD009000, 2008.

Delanoë, J., Protat, A., Bouniol, D., Heymsfield, A., Bansemer, A., and Brown, P.: The characterization of ice clouds properties from Doppler radar measurements, J. Appl. Meteorol. Climatol., 46, 1682-1698, 2007.

Deng, M. and Mace, G. G.: Cirrus cloud microphysical properties and air motion statistics using cloud radar Doppler moments: Water content, particle size, and sedimentation relationships, 
Geophys. Res. Lett., 35, L17808, doi:10.1029/2008GL035054, 2008.

Donovan, D. and Lammeren, A.: Cloud effective particle size and water content profile retrievals using combined lidar and radar observations 1. theory and examples, J. Geophys. Res., 106, 27425-27448, 2001.

Dufournet, Y.: Ice Crystal Properties Retrieval using radar spectral polarimetric measurements within ice / mixed-phase clouds, $\mathrm{PhD}$ thesis, Technische Universiteit Delft, 2010.

Heijnen, S., Ligthart, L., and Russchenberg, H.: First measurements with TARA; an S-Band transportable atmospheric radar, Phys. Chem. Earth(B), 25, 995-998, 2000.

Hogan, R. J., Illingworth, J., and Sauvageot, H.: Measuring Cystal Size Using 35- and 94-GHz Radars, J. Atmos. Oceanic Technol., 17, 27-37, 1999.

Hogan, R. J., Mittermaier, M. P., and Illingworth, A. J.: The retrieval of ice water content from radar reflectivity factor and temperature and its use in the evaluation of a mesoscale model, J. Appl. Meteorol. Climatol., 45, 301-317, 2006.

Kärcher, B. and Koop, T.: The role of organic aerosols in homogeneous ice formation, Atmos. Chem. Phys., 5, 703-714, doi:10.5194/acp-5-703-2005, 2005.

Lamb, D. and Scott, W.: Linear growth rates of ice crystals grown from the vapor phase, J. Cryst. Gr., 12, 21-31, 1972.

Lawson, R., Baker, B., Schnitt, C., and Jensen, T.: An overview of microphysical properties of arctic clouds observed in May and July 1998 during Fire Ace., J. Geophys. Res., 106, 14989-15014, 2001.

Mace, G. G., Ackerman, T. P., Minnis, P., and Young, D. F.: Cirrus layer microphysical properties derived from surface-based millimeter radar and infrared interferometer data, J. Geophys. Res., 103, 23207-23216, doi:10.1029/98JD02117, 1998.

Matrosov, S. Y., Reinking, R. F., Kropfli, R. A., Martner, B. E., and Bartram, B. W.: On the use of Radar Depolarization Ratios for Estimating Shapes of Ice Hydrometeors in Winter Clouds, J. Appl. Meteorol., 40, 479-490, 2000.

Matrosov, S. Y., Korolev, A. V., and Heymsfield, A. J.: Profiling cloud ice mass and particle characteristic size from doppler radar measurements, J. Atmos. Ocean. Tech., 19, 1003, doi:10.1175/1520-0426(2002)019;1003:PCIMAP 2 2.0.CO;2, 2002 .
Mitchell, D.: Use of mass- and area-dimensional power laws for determining precipitation particle terminal velocities, J. Atmos. Sci., 53, 1710-1723, 1996.

Mogano, C. and Lee, C.: Meteorological classification of natural snow crystals, J. Fac. Sci., 2, 321-335,1966

Protat, A., Delanoë, J., Bouniol, D., Heymsfield, A. J., Bansemer, A., and Brown, P.: Evaluation of Ice Water Content Retrievals from Cloud Radar Reflectivity and Temperature Using a Large Airborne In Situ Microphysical Database, J. Appl. Meteorol. Climatol., 46, 557-572, 2007.

Russchenberg, H.: Ground-based remote-sensing of precipitation using a multi-polarized FM-CW Doppler radar, PhD thesis, Technische Universiteit Delft, 1992.

Shupe, M. J., Kollias, P., Matrosov, S. Y., and Schneider, T. L.: Deriving Mixed-Phase Cloud Properties from Doppler Radar Spectra., J. Atmos. Oceanic Technol., 21, 660-670, 2003.

Shupe, M. J., Daniel, J., de Boer, G., Eloranta, E., Kollias, P., Long, C., Luke, E., Turner, D., and Verlinde, J.: A focus on mixedphase clouds: the status of ground-based observation methods, B. Am. Meteorol. Soc., 89, 1549-1562, 2008.

Tinel, C., Testud, J., Pelon, J., Hogan, R. J., Protat, A., Delanoeë, J., and Bouniol, D.: The retrieval of ice-cloud properties from cloud radar and lidar synergy. J. Appl. Meteorol., 44, 860-875, 2005.

Unal C.: Spectral polarimetric radar clutter suppression to enhance atmospheric echoes., J. Atmos. Oceanic Technol., 2009.

Unal, C. and Moisseev, D.: Combined Doppler and polarimetric radar measurements: Correction for spectrum aliasing and nonsimultaneous polarimetric measurements, J. Atmos. Oceanic Technol., 21, 443-455, 2004.

Wulfmeyer, V., Behrendt, A., Bauer, H.-S., Kottmeier, C., Corsmeier, U., Blyth, A., Craig, G., Schumann, U., Hagen, M., Crewell, S., Di Girolamo, P., Flamant, C., Miller, M., Montani, A., Mobbs, S., Richard, E., Rotach, M. W., Arpagaus, M., and Russchenberg H.: The Convective and Orographically-induced Precipitation Study: A Research and Development Project of the World Weather Research Program for improving quantitative precipitation forecasting in low-mountain regions., B. Am. Meteorol. Soc, 89, 1477-1486, 2008.

Wang, Z. and Sassen, K.: Cirrus Cloud Microphysical Property Retrieval Using Lidar and Radar Measurements, Part II: Midlatitude Cirrus Microphysical and Radiative Properties, J. Appl. Meteorol., 41, 218-229, 2002. 